\title{
Design of a Drug-Delivery System Based On Polyacrylamide Hydrogels. Evaluation of Structural Properties
}

\author{
L. Ferreira, ${ }^{\star}$ M. M. Vidal, ${ }^{\dagger, *}$ and M. H. Gil ${ }^{*}$ \\ Departamento de Engenharia Química, Universidade de Coimbra, Pinhal de Marrocos, 3030 Coimbra, \\ Portugal, and Escola Superior Agrária de Coimbra, 3000 Coimbra, Portugal, mariavidal10@hotmail.com
}

Received October 2, 2000. Accepted December 12, 2000

\begin{abstract}
It is well known that hydrogels can be suitable for biomedical, agricultural, and industrial applications. In particular, they have been widely used for the preparation of drug- delivery systems. The preparation and characterization of such a system should be useful for introducing students to these materials. This paper describes the preparation of polyacrylamide hydrogels having different crosslinking densities from the view of optimizing this system for acetylsalicylic acid (aspirin) release. The observations of equilibrium swelling, solute transport, and thermal analysis are related to the network structure of polyacrylamide hydrogel.
\end{abstract}

\section{Introduction}

The development of new drug-delivery systems is a subject that may be of interest to undergraduate or graduate students in the areas of chemistry, pharmacy, and materials. In this paper, we report the preparation and characterization of a hydrogel that could be used to introduce students to this area.

Hydrogels are three-dimensional polymer networks in which individual hydrophilic polymer chains are connected, either by physical association or by chemical bonds. When in contact with water, this kind of structure is able to absorb a considerable amount of the water without dissolving, showing the mechanical properties of solids and the diffusive transport properties of liquids [1-3]. Due to their high water content, hydrogels are highly amenable for use as biomaterials, that is, materials that can be in contact with tissue or biological fluids. For this reason, hydrogels have been extensively studied for biomedical applications, such as implants, soft contact lenses, and drug-delivery systems (DDS) [4-7].

A drug-delivery system must be able to control solute release over time; thus, the development of drug-delivery systems requires the control of the water content within the polymeric structure. This is one of the most important factors influencing the solute transport. In the last few years, various materials have been used for this purpose; however, hydrogels show two distinct advantages. First, drugs can easily diffuse through the hydrogels. The permeation rate can be controlled either by changing the crosslinking densities or by preparing the hydrogel with monomers of controlled hydrophilicity. Second, hydrogels may interact less strongly with the drugs than more hydrophobic materials; consequently, a larger fraction of active molecules is able to leave the hydrogel. In the case of neutral hydrogels, the diffusion mechanism is dependent on the mesh size, which is related to the crosslinking density [8].

This work describes the study of a simple DDS, based on a neutral polyacrylamide hydrogel. The hydrogels are obtained by free radical copolymerisation of the acrylamide (AAm) and the bifunctional monomer $N, N^{\prime}$-methylenebisacrylamide (MBAAm) in aqueous solution using a initiator system of potassium persulphate and $N, N, N^{\prime}, N^{\prime}$-tetramethylenediamine, $\mathrm{K}_{2} \mathrm{~S}_{2} \mathrm{O}_{8}$ /TEMED (Figure 1) [9]. For the release studies, acetylsalycilic acid (ASA, aspirin) is used as a model drug. ASA diffusion coefficients are determined to demonstrate the dependence of this parameter on the crosslink density.

\section{Theory}

Diffusion Mathematics. The flux, $J$, of a diffusing substance through the unit area of a membrane can be expressed by Fick's first law

$$
J=-D\left(\frac{\partial c}{\partial x}\right)
$$

where $\partial C / \partial x$, the concentration gradient measured normal to the membrane, is the driving force for the diffusion, and $D$ is the diffusion coefficient expressed in length ${ }^{2} \operatorname{time}^{-1}\left(\mathrm{~m}^{2} \mathrm{~s}^{-1}\right.$ in SI units). The diffusion coefficient is a constant independent of $x, c$, and the time, $t$. For these conditions, and when the concentration gradient of the permeant across the membrane varies with the time, the rate of change of permeant concentration at any point is given by Fick's second law.

$$
\frac{\partial c}{\partial t}=\frac{D \partial^{2} c}{\partial x^{2}}
$$

Several solutions of Fick's differential equation that depend on the boundary conditions were developed by Crank [10]. Equation 3 is a good approximation for the solution obtained when the surface concentration is constant at both faces of the membrane and for values of $M_{\mathrm{t}} / M_{\infty}$ less than 0.5 .

$$
M_{t} / M_{\infty}=\left(4 / l_{o} \pi^{1 / 2}\right) D^{1 / 2} t^{1 / 2}
$$

\footnotetext{
${ }^{*}$ Address correspondence to this author.

${ }^{\dagger}$ Escola Superior Agrária de Coimbra

¥University of Coimbra
} 


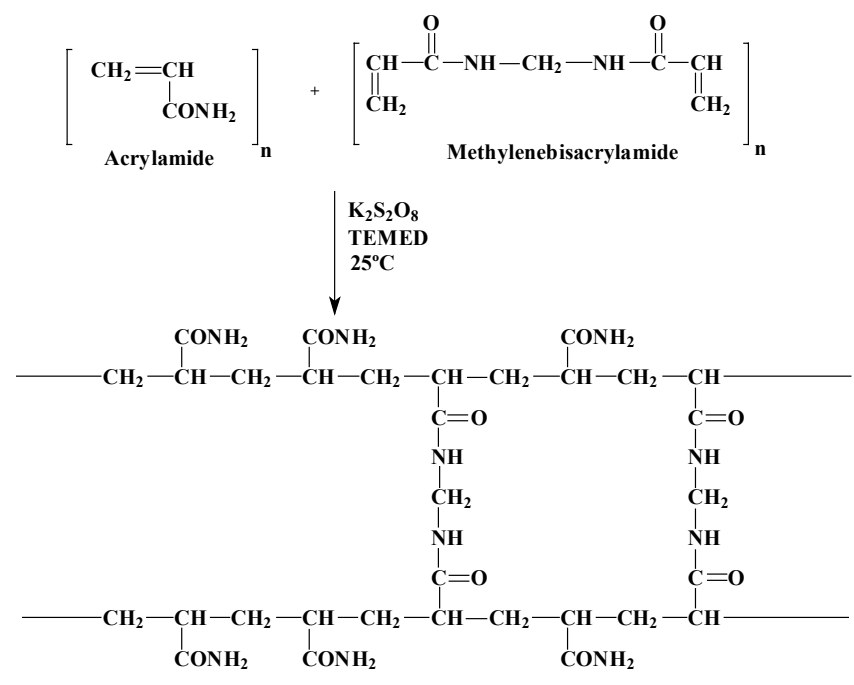

Polyacryla mide Hydrogel

Figure 1. Schematic pathway for the synthesis of polyacrylamide hydrogels.

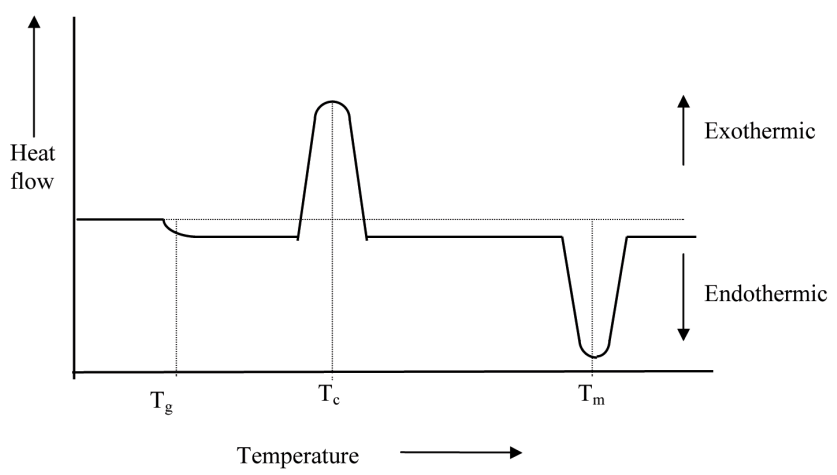

Figure 2. Schematic representation of a DSC curve related to a semicrystalline polymer showing the glass transition temperature $\left(T_{\mathrm{g}}\right)$, crystallization temperature $\left(T_{\mathrm{c}}\right)$ and melting temperature $\left(T_{\mathrm{m}}\right)$.

Table 1. MBAAm Concentration Variation for Crosslinked Polyacrylamide Hydrogels

\begin{tabular}{cc}
\hline Hydrogel & MBAAm $(\mathrm{mg})^{\mathrm{a}}$ \\
\hline 1 & 5 \\
2 & 10 \\
3 & 20 \\
4 & 30 \\
5 & 40 \\
6 & 60 \\
7 & 100 \\
\hline
\end{tabular}

${ }^{\mathrm{a}}$ Besides MBAAm, the feed composition of the gels was: $0.2 \mathrm{~g} \mathrm{AAm}, 2$ mg $\mathrm{K}_{2} \mathrm{~S}_{2} \mathrm{O}_{8}, 40 \mu \mathrm{L}$ TEMED, and $1 \mathrm{~mL}$ of $\mathrm{H}_{2} \mathrm{O}$ or ASA aqueous solution $(2.5 \mathrm{mg} / \mathrm{mL})$.

Here, $l_{\mathrm{o}}$ is the thickness of the hydrogel, $D$ is the diffusion coefficient of the solute, $M_{\mathrm{t}}$ is the amount of solute released at time $t$, and $M_{\infty}$ is the total amount of solute in the hydrogel. When fractional release, $M_{\mathrm{t}} / M_{\infty}$, is linear with the square root of time for values of $M_{\mathrm{t}} / M_{\infty}$ less than 0.5 , the release-curve profiles match Fick's law, allowing the determination of diffusion coefficients. Thus, the diffusion coefficient can be experimentally obtained from the slope $(m)$ of the curve, given by eq 4

$$
m=\left(4 / l_{o} \pi^{1 / 2}\right) D^{1 / 2}
$$

Thermal Analysis by Differential Scanning Calorimetry. Thermal analysis is defined by ICTA (the International Confederation for Thermal Analysis) as a term covering a group of techniques in which a physical property of a substance and/or its reaction products are measured as a function of temperature [11]. Differential scanning calorimetry (DSC) is a technique that measures the energy difference between a sample and the reference cell as a function of time when they are submitted to a heating program. The technique is useful for polymers, particularly hydrogels, because the structural changes accompanied by energetic effects can be followed in DSC curves. A typical thermogram for a semicrystalline polymer is shown in Figure 2 where both firstand second-order transitions are shown.

The first-order transition, which is related to crystallization and melting processes, presents a well-defined peak whose respective area is proportional to the enthalpy variation $(\Delta H)$, allowing the determination of the crystalline degree of the polymer. The second-order transition, related to the glasstransition temperature, involves a variation of the heat capacity and can be determined from the inflexion point [12].

\section{Experimental Procedure}

Caution: Due to the toxic nature of the acrylamide [13], skin contact should be absolutely avoided. Gloves and protective mask should be used. Polyacrylamide gels are not toxic and no special care is needed in their handling.

Synthesis of the Hydrogels (Laboratory time: $4 \mathrm{hrs}$ ). Two different sets of polyacrylamide hydrogels are prepared. The two sets differ in that one set contains acetylsalicylic acid and the other does not. The different hydrogels within each set have different percentages of MBAAm. Both of the mono- and bifunctional monomers are dissolved in $1 \mathrm{~mL}$ of water (protocol A) or in $1 \mathrm{~mL}$ of acetylsalicylic acid solution (ASA, $2.5 \mathrm{mg} / \mathrm{mL}$ ) (protocol B), at $50{ }^{\circ} \mathrm{C}$ in a water bath. The solutions are cooled and $2 \mathrm{mg}$ of initiator $\left(\mathrm{K}_{2} \mathrm{~S}_{2} \mathrm{O}_{8}\right)$ is added. After complete dissolution, $40 \mu \mathrm{L}$ of TEMED is added. The contents are vigorously stirred in order to ensure a homogeneous solution, poured onto a glass plate $(2 \mathrm{~cm} \times 2 \mathrm{~cm})$, and allowed to polymerize at room temperature. After $15 \mathrm{~min}$, the hydrogels are completely formed. To prepare the ASA solution, $0.5 \mathrm{~g}$ of ASA is dissolved in $10 \mathrm{~mL}$ of $1 \mathrm{M} \mathrm{NaOH}$, and the solution is brought to $200 \mathrm{~mL}$ with distilled water.

The reaction mixtures of each hydrogel system are shown in Table 1.

Swelling Measurements (Laboratory time: 4 hr). Hydrogels without ASA (protocol A) are thoroughly washed with distilled water to remove unreacted monomer and crosslinking agent (during 3 to 5 days). Before changing the water everyday, the hydrogels are removed from the water, blotted with filter paper to remove the water from the sample surface, and their mass is determined. This procedure is repeated until the hydrogels swell to equilibrium, which is taken as the time at which their weight stops increasing. The hydrogels are then dried at room temperature under vacuum in the presence of phosphorous pentoxide $\left(\mathrm{P}_{2} \mathrm{O}_{5}\right)$, to constant weight.

The equilibrium water content (EWC) was calculated using eq 5 . $M_{\mathrm{s}}$ is the mass of the swollen gel and $M_{\mathrm{d}}$ is the mass of dry gel.

$$
E W C=\left[\left(M_{s}-M_{d}\right) / M_{s}\right] \times 100
$$

Determination of the ASA Release from Polyacrylamide Hydrogels (Laboratory time: $4 \mathbf{h r}$ ). Hydrogels containing ASA 


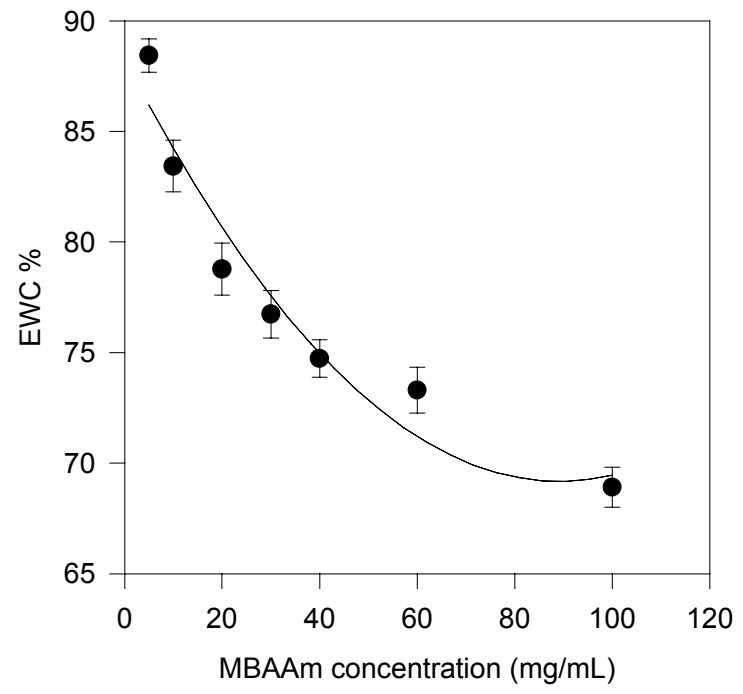

Figure 3. Variation in equilibrium water content for polyacrylamide hydrogels with different MBAAm content at $25^{\circ} \mathrm{C}$.

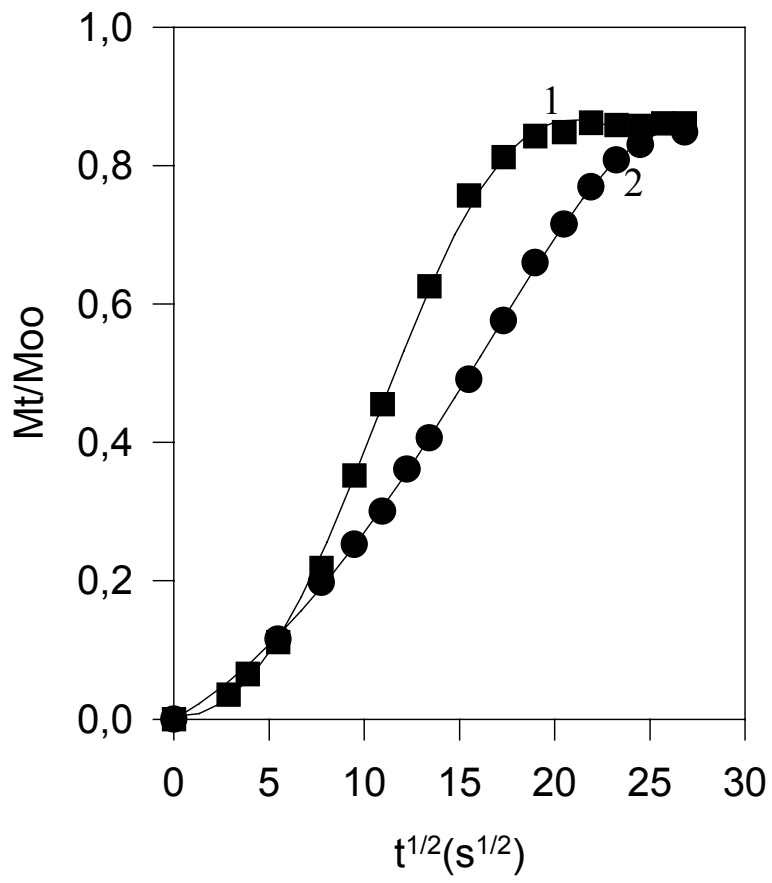

Figure 4. Normalized solute release, $\mathrm{Mt} / \mathrm{M} \infty$, from polyacrylamide hydrogels versus $t^{1 / 2}$ (experimental data). Release in water of ASA from polyacrylamide hydrogels with $5 \mathrm{mg}$ (1) and $100 \mathrm{mg}$ (2) of MBAAm.

Table 2. Diffusion Coefficients for Polyacrylamide Hydrogels

\begin{tabular}{ccc}
\hline Hydrogel & Thickness $(\mathrm{cm})$ & Diffusion coefficient $(D)\left(\mathrm{cm}^{2} \mathrm{~s}^{-1}\right)$ \\
\hline 1 & 0.019 & $1.2 \times 10^{-7}$ \\
7 & 0.065 & $8.5 \times 10^{-8}$ \\
\hline
\end{tabular}

(protocol B) are placed in a 98\% relative humidity atmosphere. This is created by a saturated solution of copper sulfate pentahydrate in a closed chamber. Exposure continues until equilibrium swollen states are reached. Each of the hydrogels is immersed in fresh distilled water $(50 \mathrm{~mL})$ with magnetic stirring for $15 \mathrm{~min}$ at $37^{\circ} \mathrm{C}$. The absorbance values of these solutions, at $300 \mathrm{~nm}$, are determined every minute. These absorbance measurements are then converted to ASA concentrations using an ASA calibration curve.

Note: release experiments can also be done in a phosphate buffer to eliminate the effect of distilled water $\mathrm{pH}$ differences and to better mimic the biological environment.

Differential Scanning Calorimetry (Laboratory time: $4 \mathrm{hr}$ ). Differential scanning calorimetry thermograms are obtained. We use a Polymer Laboratories DSC system. The hydrogels are dried at room temperature under vacuum and in the presence of $\mathrm{P}_{2} \mathrm{O}_{5}$, until constant weight is obtained. Then, samples with mass values between 2 and 5 $\mathrm{mg}$ are sealed into an aluminum pan. An empty aluminum sealed pan was used as a reference. The temperature is raised from $25^{\circ} \mathrm{C}$ to 250 ${ }^{\circ} \mathrm{C}$ at a heating rate of $10{ }^{\circ} \mathrm{C} / \mathrm{min}$ under a nitrogen atmosphere (flow, $\left.10 \mathrm{~cm}^{3} / \mathrm{min}\right)$.

\section{Results and Discussion}

Swelling Measurements. Observation of the hydrogels 1-7, made according to protocol $\mathrm{A}$, shows that both the mechanical behavior and the transparency are influenced by the MBAAm content. At an MBAAm concentration up to $20 \mathrm{mg}$, the gels obtained are transparent and mechanically weak. The mechanical strength and opacity increases with increasing MBAAm content. The EWC dependence on MBAAm content is shown in Figure 3. It can be seen that the EWC decreases with increased crosslinking density, particularly, for the first three hydrogels (1-3).

Determination of the ASA Release from Polyacrylamide Hydrogels. The release of ASA, a hydrophilic solute with a molecular weight of $180 \mathrm{~g} \mathrm{~mol}^{-1}$, is studied as a function of network composition. For this purpose the UV peak of ASA at $300 \mathrm{~nm}$ is used to monitor the solute release. At this wavelength and for the total volume used $(50 \mathrm{~mL})$, the absorbance contribution of unreacted monomers (AAm and MBAAm) due to their diffusion from the hydrogel is not important. The diffusion coefficients are obtained by eq 3 . Figure 4 shows the release of ASA from hydrogels (1 and 7) for two different degrees of crosslinking, at $37^{\circ} \mathrm{C}$ into distilled water. As shown in this figure, after an initial lag phase $\left(t^{1 / 2}<\right.$ 6 , corresponding to $\left.M_{\mathrm{t}} / M_{\infty}<0.1\right)$ [14], the experimental release curves $\left(0.1<M_{\mathrm{t}} / M_{\infty}<0.5\right)$ can be considered linear, allowing the experimental determination of the diffusion coefficient from the straight-line slopes $(m)$ given by eq 4 . The diffusion coefficients calculated from these release curves are given in Table 2. As can be seen from the table, the release from gel 1 is more rapid than from gel 7 . The highly swollen gel has a diffusion coefficient of $1.2 \times 10^{-7} \mathrm{~cm}^{2} \mathrm{~s}^{-1}$ and a $t_{50 \%}$ of $132 \mathrm{~s}$, while the gel with a low degree of swelling has a diffusion coefficient of $8.5 \times 10^{-8} \mathrm{~cm}^{2} \mathrm{~s}^{-1}$ and a $t_{50 \%}$ of $240 \mathrm{~s}$. The symbol $t_{50 \%}$ is the time required to release $50 \%$ of the solute.

Differential Scanning Calorimetry. Figure 5 shows the heating DSC curves obtained for polyacrylamide hydrogels prepared without ASA (hydrogels 1, 3, 5, and 7). From these thermograms, the glass-transition temperature $\left(T_{\mathrm{g}}\right)$, related to a variation in the heat capacity; the melting point temperature $\left(T_{\mathrm{m}}\right)$ for which the melting of the crystalline phase occurs; and the melting enthalpy $\left(\Delta H_{\mathrm{f}}\right)$, the energy required to transform $1 \mathrm{~g}$ of a crystalline material to $1 \mathrm{~g}$ of an amorphous material, have been calculated. The values are shown in Table 3.

The area of the endothermic peak, related to the fusion of the crystalline phase, gives an estimate of $\Delta H_{\mathrm{f}}$. The results 


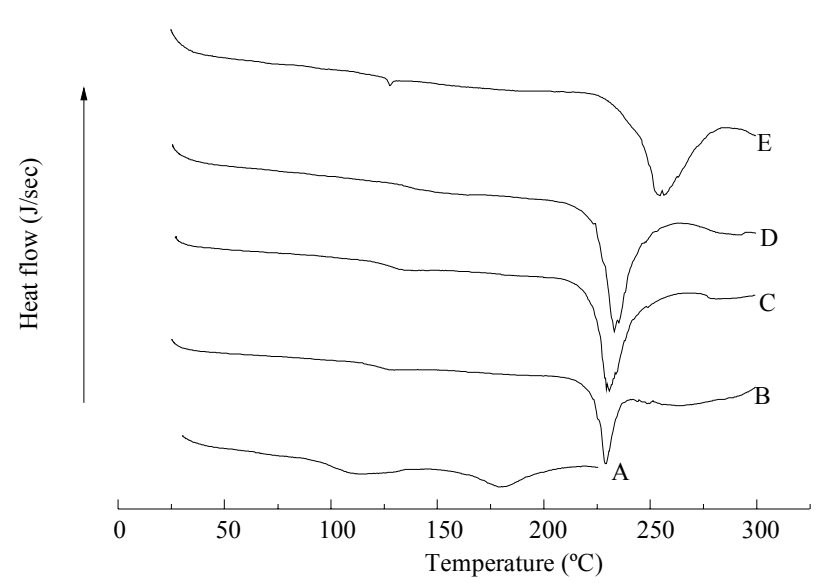

Figure 5. DSC curves for polyacrylamide hydrogels prepared with different MBAAm concentrations: (A) polyacrylamide, (B) Hydrogel 1, (C) Hydrogel 3, (D) Hydrogel 5, and (E) Hydrogel 7.

Table 3. Thermal Parameters Obtained by DSC for Different Hydrogels

\begin{tabular}{cccc}
\hline Sample & $T_{\mathrm{g}}\left({ }^{\circ} \mathrm{C}\right)$ & $T_{\mathrm{m}}\left({ }^{\circ} \mathrm{C}\right)$ & $\Delta H_{\mathrm{m}}(\mathrm{J} / \mathrm{mg}) \times 10^{-2}$ \\
\hline polyacrylamide & 101.4 & 179.9 & 3.8 \\
hydrogel 1 & 121.1 & 228.9 & 6.4 \\
hydrogel 3 & 126.9 & 230.9 & 1.4 \\
hydrogel 5 & 139.7 & 233.3 & 1.4 \\
hydrogel 7 & $-\mathrm{a}$ & 254.7 & 1.2 \\
\hline
\end{tabular}

${ }^{-a}$ Not determined

show that $\Delta H_{\mathrm{m}}$ increases gradually with an increase in the MBAAm content up to $30 \mathrm{mg}$. This increase reflects the increase of the crystalline zone in the structure of gel. On the other hand, the stabilization of $\Delta H_{\mathrm{f}}$ for the highly crosslinked hydrogels $\quad([\mathrm{MBAAm}]=30 \mathrm{mg}) \quad$ indicates that the crystallization of the hydrogels reaches a plateau and becomes independent of further crosslinking. The increase of $T_{\mathrm{g}}$ with increasing crosslink density and the disappearance of this transition for the most highly crosslinked gel (gel 7) can be ascribed to the gradual immobilization of chain segments in the amorphous parts of the gel. This finding is supported by EWC studies and by solute diffusion measurements.

These results suggest gel structure development according to the Silbeberg model [15]. During the polymerization process, the gel structure, incorporating both monofunctional and bifunctional monomers, becomes more complex, creating diffusion problems. The reaction proceeds with loop formation between two activated monomer units incorporated in the chain. The interaction of these loops with the formed chain, immobilizing individual chains from the amorphous phase, increases $T_{\mathrm{g}}$ and $T_{\mathrm{m}}$ and favors crystallization, increasing $\Delta H_{\mathrm{f}}$.

\section{Conclusions}

In this work we investigated the structural characterization of polyacrylamide hydrogels used as drug delivery systems.
First, we observed high levels of water content, characteristic of hydrogels. Second, from thermal behavior studies, we are able to say that the crosslinking-agent concentration influences the crystallinity, and the more crystalline the structure the lower the EWC values. So, we observed a direct dependence of the water content on the crosslinking-agent concentration. The dependence of ASA diffusion coefficients on the water content within the gel structure was also observed, indicating that crosslink density offers a simple way of controlling the diffusion properties of the polyacrylamide hydrogels. These gels have advantages due to their low cost, the simplicity of their preparation, and the ability to control their diffusion properties. This makes them very attractive materials for biomedical and agricultural applications.

This experiment includes concepts like polymerization, crosslink density, and controlled release, topics which should be emphasized to students in the areas of chemistry, pharmacy and materials science. These concepts are also valuable for students with interests in the life sciences.

\section{References and Notes}

1. Kulicke, W. M.; Nottelman, H. In Polymers in Aqueous Media; Glass, J. E., Ed.; ACS Symposium Series No. 223; American Chemical Society: Washington, DC, 1989, p 15.

2. Garner, C. M.; Nething, M.; Nguyen, P. J. Chem. Educ. 1997, 74 (1), 95-96.

3. Buchholz, F. L. J.Chem. Educ. 1996, 73 (6), 512-515.

4. Ratner, B. D.; Hoffman, A. S. In Hydrogels for Medical and Related Applications; Andrade, J. D., Ed.; ACS Symposium Series No. 31; Washington, DC, 1976, p 1.

5. Geaskens, G.; Soukrati, A. Eur. Polym. J. 2000, 36 (8), 1537-1546.

6. Peppas, N. A. In Biomaterials Science: An introduction to materials in medicine; Ratner, B. D.; Hoffman, A. S.; Schoen, F. J.; Lemon, J. E., Eds.; Academic Press, 1996, pp 60-64.

7. Peppas, N. A.; Lustig, S. R. In Hydrogels in Medicine and Pharmacy; Peppas, N. A., Ed.; Boca Raton, 1986, Vol. 1, pp 57-83.

8. Peppas, N. A.; Klier, J. J .Controlled Release 1991, 16, 203-214.

9. Bikales, N .M. In Vinyl and Diene Monomers; Leonard, E. C., Ed.; Wiley Interscience: New York, 1970, Part I, p 81.

10. Crank, J. In The Mathematics of Diffusion; Crank, J., Ed; Oxford: Clarendon Press, 1970.

11. Mackenzie, R. C. Pure Appl. Chem. 1985, 57, 1737.

12. Gray, A. P. Thermochim. Acta 1970, 1, 563-579.

13. Information about the toxic nature of acrylamide is available from The International Labor Organization. http://www.ilo.org (accessed Jan 2001).

14. The slight deviation from linearity for initial solute release $\left(M_{\mathrm{t}} / M_{\infty}<\right.$ 0.1) can be ascribed to several factors already described in the literature and does not mean that the process is not diffusion controlled, that is, a Fickian process. (See, for instance, Gehrke, S. H.; Biren, D., Hopkins, J. J. ; In Polymer Biomaterials in Solution, as Interfaces and as Solids; Cooper, S. L.; Bamford, C. H.; Tsuruta, T., Eds.; VSP: Utrecht, Netherlands, 1995, pp 1043-1058).

15. Silberberg, A. In Polymers in Aqueous Media; Glass, J. E. Ed.; ACS Symposium Series No. 223; American Chemical Society: Washington, 1989, p 3. 